\title{
The Effects of Gaussian Weighting Errors in Hybrid SC/MRC Combiners
}

\author{
A. Annamalai ${ }^{1}$ and C. Tellambura ${ }^{2}$
}

1. Bradley Department of Electrical and Computer Engineering, Virginia Tech, $206 \mathrm{~N}$. Washington St., Suite 400 Alexandria, VA 22314, USA, Tel: +1-703-535-3459, Fax: +1-703-518-8085, E-mail: annamalai@vt.edu

2. School of Computer Science and Software Engineering, Monash University, Clayton, Victoria 3168, Australia Tel: +1-613-9905-3296, Fax: +1-613-9905-3402, E-mail: chintha@dgs.monash.edu.au

\begin{abstract}
The paper examines the impact of Gaussian distributed weighting errors (in the channel gain estimates used for coherent combination) on the statistics of the output of hybrid selection/maximal-ratio (SC/MRC) receiver as well as the degradation of the average symbol error rate (ASER) performance from the ideal case. New expressions for the probability density function (PDF), cumulative distribution function (CDF) and moment generating function (MGF) of the coherent hybrid SC/MRC combiner output signal-to-noise ratio (SNR) are derived. The MGF is then used to derive exact closed form ASER formulas for binary and M-ary modulations employing nonideal hybrid SC/MRC receiver in Rayleigh fading. Results for both SC and MRC are obtained as limiting cases. The effect of the weighting errors on the outage rate of error probability and the average combined SNR are also investigated. These analytical results provide some insights into the trade-off between diversity gain and combination losses with the increasing order of diversity branches in an energy-sharing communication system.
\end{abstract}

\section{INTRODUCTION}

Recently, hybrid SC/MRC diversity scheme has received considerable attention in the literature owing to its ability to counter balance the detrimental effects of deep fades on wireless channels, while achieving a good compromise between the receiver performance and the implementation complexity (fewer electronics as well as lower power consumption) [1]-[9]. In an $M / L-S C / M R C$ system, the receiver selects the $M$ strongest branches of $L$ diversity branches and then coherently combine them in a MRC combiner to produce the decision statistic. The study of hybrid SC/MRC receiver is important both from a practical viewpoint and theoretical standpoint because this model encapsulates both $\mathrm{SC}(M=1)$ and $\operatorname{MRC}(M=L)$ receiver performance as limiting cases.

In all the above analyses, the channel gain estimates are assumed to be noiseless. In fact, only a few published results on imperfect coherent combining are available. Five notable contributions are [10]-[14]. In [10], Bello and Nelin first showed that decorrelation of the pilot from the data signal separated in frequency to be Gaussian distributed and then derived the average bit-error rate (ABER) expressions for both coherent and noncoherent detection of binary orthogonal signals employing nonideal MRC receiver in Rayleigh fading. In [11], the investigation into the effects of weighting errors on ABER was confined to the development of bounds on the reduction of average out- put SNR for a fixed amplitude error or a fixed phase error in the weighting factor. Proakis [12] derived ABER expressions for MPSK signalling scheme in Rayleigh and Rician channels when an ad hoc estimator eased on pilot signal or clairvoyant information from the data signal is used to determine the channel weights. In [13], Gans modelled the channel estimation errors in MRC as being complex Gaussian and subsequently derived the PDF and CDF of the combiner output SNR in Rayleigh fading. The Gaussian errors may be due to inaccurate signal estimation (e.g. clairvoyant estimator used in [12]) or due to decorrelation of the pilot from the signal because of too large frequency or time separation. More recently, [14] showed that the ABER for arbitrary modulation formats employing nonideal MRC can be written in a canonical form, i.e., as a weighted sum of $L$ terms of ideal ABER employing MRC with increasing diversity orders.

The major contributions of this paper include: (a) derivation of PDF, CDF and MGF of the hybrid $M / L-S C / M R C$ combiner output SNR taking into account of the Gaussian distributed weighting errors in the channel gain estimates; (b) derivation of closed-form ASER formulas for a broad class of modulation formats in conjunction with nonideal MRC; and (c) derivation of analytical expressions for computing the outage rate of error probability for several common modulation formats and the average combiner output SNR. To the best of the authors' knowledge, study of the effect of Gaussian weighting errors on the PDF, CDF and MGF of the hybrid SC/MRC combiner output SNR is not available in the literature. The investigation into the effect of Gaussian errors in SC receiver also appears to be new. Similarly, closed-form expressions for the ASER of arbitrary modulations formats in conjunction with SC/MRC receiver and their corresponding outage rate have not been reported previously. It is also not very clear if the complexity of combiner and the combination losses (due to weighting errors in the gain estimates) tend to outweigh the benefits obtained from additional diversity branches in an energy sharing communication system. Insights to this are provided in our forthcoming paper [15].

\section{HYBRID SC/MRC COMBINER OUTPUT STATISTICS} In this section, we will summarize our results for the PDF, $\mathrm{CDF}$ and MGF of nonideal $M / L-S C / M R C$ combiner output SNR on i.i.d Rayleigh fading channels by assuming that the 
weighting error is complex Gaussian. Details of our derivations can be found in [15].

Following the mathematical procedure in [13], it is possible to show (after a lengthy derivation and simplifications) that the PDF of the MIL-SC/MRC combiner output SNR with Gaussian distributed weighting errors is given by

$$
\begin{aligned}
& p_{\gamma}(\gamma)=\sum_{l=0}^{L-M} \exp \left(-\frac{\gamma}{\Lambda_{l}}\right) \sum_{k=1}^{D_{l}} \frac{\mu_{t}^{\left(D_{l}-k\right)}}{\Lambda_{l}^{k}}\left[\bar{\gamma}\left(1-\rho^{2}\right)\right]^{k-1} \\
& \times \sum_{n=0}^{k-1} \frac{1}{n !}\left(\begin{array}{c}
k-1 \\
n
\end{array}\right)\left[\frac{M \rho^{2} \gamma}{\Lambda_{1}\left(1-\rho^{2}\right)(l+M)}\right]^{n}
\end{aligned}
$$

where $\Lambda_{t}=\bar{\gamma}\left[\frac{M+l\left(1-\rho^{2}\right)}{l+M}\right], D_{l}=\left\{\begin{array}{c}M \text { for } l=0 \\ 1 \text { for } l \neq 0\end{array}, \rho\right.$ denotes the correlation between the actual complex channel gains and their estimates, $\bar{\gamma}$ is the average SNR/symbol per branch, and the coefficients $\mu_{1}^{(0)}$ and $\mu_{0}^{(1 /-k)}$ (for $k<M$ ) may be computed as

$$
\begin{aligned}
\mu_{l}^{(0)} & =\prod_{i=0, i \neq l}^{L-M}\left(\frac{i+M}{i-l}\right)^{D_{i}} \\
& =\left\{\begin{array}{cc}
\left(\begin{array}{c}
L \\
M
\end{array}\right) & \text { for } l=0 \\
(-1)^{M+l-1}\left(\frac{M}{l+M}\right)\left(\frac{M}{l}\right)^{M /-1}\left(\begin{array}{c}
L \\
M
\end{array}\right)\left(\begin{array}{c}
L-M \\
l
\end{array}\right) \text { for } l \neq 0
\end{array}\right. \\
\mu_{0}^{(M-k)} & =\left\{\begin{array}{cc}
\sum_{i=1}^{L-M}(-1)^{i+M-k-1}\left(\begin{array}{c}
L \\
M
\end{array}\right)\left(\begin{array}{c}
L-M \\
i
\end{array}\right)\left(\frac{M}{i}\right)^{M-k} & \text { if } M<L \\
0 & \text { if } M=L
\end{array}\right.
\end{aligned}
$$

Then, the CDF of hybrid SC/MRC combiner output SNR with Gaussian distributed weighting errors can be readily shown as

$$
\begin{aligned}
F_{\gamma}(\gamma)=1- & \exp \left(-\frac{\gamma}{\Lambda}\right)_{i=0}^{L-k} \sum_{k=1}^{D_{l}}\left[\frac{\dot{\gamma}\left(1-\rho^{2}\right)}{\Lambda_{l}}\right]^{k-1} \mu_{l}^{\left(D_{j}-k\right)} \sum_{n=01}^{k-1}\left(\begin{array}{c}
k-1 \\
n
\end{array}\right) \\
& \times\left[\frac{M \rho^{2}}{\left(1-\rho^{2}\right)(l+M)}\right]_{w=0}^{n} \sum_{w !}^{n} \frac{1}{w !}\left(\frac{\gamma}{\Lambda}\right)^{w}
\end{aligned}
$$

Taking Laplace transform of the PDF (1), we get the MGF of the output SNR:

$$
\begin{aligned}
\phi_{\gamma}(s) & =\sum_{i=0}^{L-\lambda l} \sum_{k=1} \mu_{l}^{\left(D_{l}-k\right)}\left[\frac{\bar{\gamma}\left(1-\rho^{2}\right)}{\Lambda_{l}}\right]^{k-1} \sum_{n=0}^{k-1}\left(\begin{array}{c}
k-1 \\
n
\end{array}\right) \\
& \times\left[\frac{M \rho^{2}}{\left(1-\rho^{2}\right)(l+M)}\right]^{n} \frac{1}{\left(1+s \Lambda_{l}\right)^{n+1}}
\end{aligned}
$$

To the best of the authors' knowledge, the above results $((1),(4)$ and (5)) are new and generalizes the existing results on hybrid SC/MRC output SNR statistic by considering the impact of Gaussian distributed weighting errors on a variety of receiver structures. The applications of these statistics for characterizing the performance of diversity systems in terms of ASER, outage rate of error probability and average combined SNR are presented in Sections III and IV. These results are useful for to gain insights into the design of a two dimensional $\mathrm{SC} / \mathrm{MRC}$ receiver (i.e., combined multipath and antenna diversity that are usually implemented at the CDMA base-stations), or an hybrid antenna array (for millimeter-wave broadband indoor wireless communications), or a reduced complexity rake receiver structure (for wideband CDMA systems), or a simple packet combining scheme (for increasing throughput of existing wireless data networks), to name a few.

\section{AVERAGE SYMBOL ERROR RATES}

In this section, we will show that it is possible to derive ASER expressions (in closed-form) for arbitrary two dimensional M-ary signals with polygonal decision regions, M-ary DPSK and $\pi / 4$-DQPSK modulation schemes among others, in conjunction with nonideal $\mathrm{SC} / \mathrm{MRC}$ receiver, on i.i.d. Rayleigh fading channels. The key to our derivation is the application of the MGF technique (originally described in [16] for BPSK, M-ary PSK and M-ary DPSK schemes with diversity reception) and the availability of solutions to three generic trigonometric integrals provided in [17]. In order to be concise, we only summarize the final results below. Nevertheless, it should be pointed out that these expressions, in fact, can be obtained at once by inspecting only the modulation related parameters in the conditional error probability (i.e., error probability in an AWN channel) when expressed in a desirable exponential form or by inspecting the arguments of MGF and the integration limits. Readers are referred to [17] for further details on this approach.

By applying the MGF technique, we know that the ASER for a broad class of binary and M-ary modulations employing an SC/MRC receiver with Gaussian weighting errors can be expressed in terms of only the MGF given by (5). For instance, the average SER performance of an arbitrary two-dimensional signal constellations may be computed as

$$
\begin{aligned}
\bar{P}_{e}= & \frac{1}{2 \pi} \cdot \sum_{z=1}^{S} W_{z} \int_{0}^{\eta_{z}}=\phi_{r}\left[\frac{a_{z} \sin ^{2}\left(\varphi_{z}\right)}{\sin ^{2}\left(\theta+\varphi_{z}\right)}\right] d \theta \\
= & \sum_{l=0}^{L-1} \sum_{k=1}^{D_{1}} \mu_{i}^{\left(D_{z}-k\right)}\left[\frac{\tilde{\gamma}\left(1-\rho^{2}\right)}{\Lambda_{l}}\right]^{h-1 k-1} \sum_{n=0}^{k-1}\left(\begin{array}{c}
k-1 \\
n
\end{array}\right)\left[\frac{M \rho^{2}}{\left(1-\rho^{2}\right)(l+M)}\right]^{n} \\
& \times \frac{1}{2 \pi} \sum_{z=1}^{S} W_{z} \int_{0}^{n_{z}}\left[1+\frac{\Lambda_{1} a_{z} \sin ^{2}\left(\varphi_{z}\right)}{\sin ^{2}\left(\theta+\varphi_{z}\right)}\right]^{-(n+1)} d \theta
\end{aligned}
$$

where $S$ is the total number of signal points or decision subregions, $W_{z}$ is the a priori probability of the symbol to which subregion $z$ corresponds, $a_{z}, \eta_{z}$ and $\varphi_{z}$ are coefficients (constants) relating to decision subregion $z$.

Recognizing that the integral in (6) has a known closed form solution [17], (6) can be computed conveniently as

$$
\begin{aligned}
\bar{P}_{e}= & \sum_{i=0}^{L-k} \sum_{k=1}^{D_{t}} \mu_{l}^{\left(D_{t}-k\right)}\left[\frac{\bar{\gamma}\left(1-\rho^{2}\right)}{\Lambda_{l}}\right]^{k-1} \sum_{n=0}^{k-1}\left(\begin{array}{c}
k-1 \\
n
\end{array}\right)\left[\frac{M \rho^{2}}{\left(1-\rho^{2}\right)(l+M)}\right]^{\prime \prime} \\
& \times \frac{1}{2 \pi} \sum_{z=1}^{s} W_{z} I_{S}\left[\varphi_{z}, \eta_{z}+\varphi_{z}, \Lambda_{l} a_{z} \sin ^{2} \varphi_{z}, n+1\right]
\end{aligned}
$$

where

$$
\begin{aligned}
& I_{S}\left(\theta_{L}, \theta_{i}, a, b\right)=\left(\theta_{L}-\theta_{L}\right)+\sum_{n=1}^{b}(-1)^{n} \sum_{j=0}^{n-1} \frac{a^{n-r-1 / 2}}{(1+a)^{n-1 / 2}}\left(\begin{array}{c}
b \\
n
\end{array}\right)\left(\begin{array}{c}
n-1 \\
r
\end{array}\right) \\
& \quad \times P\left(\sqrt{\frac{1+a}{a}} \tan \theta_{L}, \sqrt{\frac{1+a}{a}} \tan \theta_{L}, 1+r\right), \text { for integer } b \geq 1
\end{aligned}
$$




$$
\begin{gathered}
P(a, b, n)=\frac{(2 n-3) ! !}{2^{n-1}(n-1) !}\left\{\tan ^{-1} b-\tan ^{-1} a\right. \\
\left.+\sum_{k=1}^{n-1} \frac{2^{n-1-k}(n-k-1) !}{(2 n-2 k-1) ! !}\left[\frac{b}{\left(1+b^{2}\right)^{n-k}}-\frac{a}{\left(1+a^{2}\right)^{n-k}}\right]\right\}
\end{gathered}
$$

for integer $n \geq 1$, with notation $(2 n-3) ! !=1 \cdot 3 \ldots(2 n-3)$ and $(n-1) !=1 \cdot 2 \cdot 3 \ldots(n-1)$.

Similarly, closed-form ASER expressions for coherent binary phase shift keying (BPSK), coherent binary frequency shift keying (CFSK), noncoherent binary FSK (NCFSK), binary differential PSK (DPSK), quadrature PSK (QPSK), $M_{c}$-ary square quadrature amplitude modulation $\left(M_{c}-\right.$ QAM), star-QAM, $M_{c}$-ary PSK $\left(M_{c}-\right.$ PSK), $M_{c}$-ary DPSK $\left(M_{c}\right.$-DPSK), noncoherent $M_{c}$-ary FSK $\left(M_{c}\right.$-FSK) and DQPSK with Gray coding in conjunction with $M / L-\mathrm{SC} / \mathrm{MRC}$ receiver can be derived in a similar fashion. These results are summarized below:

\section{A. BPSK, CFSK, QPSK and $M_{\mathrm{r}}-P S K$}

$$
\begin{aligned}
\bar{P}_{e}=\frac{1}{\pi} \sum_{l=0}^{L-1} \sum_{k=1}^{D_{l}} \mu_{l}^{\left(D_{l}-k\right)}\left[\frac{\bar{\gamma}\left(1-\rho^{2}\right)}{\Lambda_{l}}\right]^{k-1} \sum_{n=0}^{k-1}\left[\frac{M \rho^{2}}{\left(1-\rho^{2}\right)(l+M)}\right]^{n} \\
\times\left(\begin{array}{c}
k-1 \\
n
\end{array}\right) I_{S}\left[0, \theta_{H}, \zeta \Lambda_{l}, n+1\right]
\end{aligned}
$$

where the coefficients $\theta_{H}$ and $\zeta$ for the four different modulation formats are listed in the table below and $M_{c}$ denotes the alphabet size of $M$-ary signal constellations.

\begin{tabular}{l|l|l}
\hline Modulation & $\theta_{H}$ & $\zeta$ \\
\hline BPSK & $\pi / 2$ & 1 \\
\hline BFSK & $\pi / 2$ & $1 / 2$ \\
\hline QPSK & $3 \pi / 4$ & $1 / 2$ \\
\hline$M_{c}-$ PSK & $\pi-\pi / M_{c}$ & $\sin ^{2}\left(\pi / M_{c}\right)$ \\
\hline
\end{tabular}

\section{B. NCFSK and DPSK}

$$
\begin{aligned}
\vec{P}_{e} & =\frac{1}{2} \phi_{\gamma}(a) \\
& =\sum_{l=0}^{L-1 /} \sum_{k=1}^{D_{l}} \frac{\mu_{t}^{\left(D_{t}-k\right)}\left(\bar{\gamma} / \Lambda_{l}\right)^{k-1}}{2\left(1+a \Lambda_{l}\right)}\left[1-\rho^{2}+\frac{M \rho^{2}}{(l+M)\left(1+a \Lambda_{t}\right)}\right]^{k-1}
\end{aligned}
$$

where $a=1$ for DPSK and $a=1 / 2$ for NCFSK.

C. $M_{c}-D P S K$, noncoherent detection of equiprobable correlated binary signals and $\pi / 4-D Q P S K$

$$
\begin{aligned}
\bar{P}_{r}= & \sum_{l=0}^{L-k} \sum_{k=1}^{D_{l}} \mu_{l}^{\left(D_{l}-k\right)}\left[\frac{\bar{\gamma}\left(1-\rho^{2}\right)}{\Lambda_{l}}\right]^{k-1} \sum_{n=0}^{k-1}\left[\frac{M \rho^{2}}{\left(1-\rho^{2}\right)(l+M)}\right]^{\prime \prime} \\
& \quad \times\left(\begin{array}{c}
k-1 \\
n
\end{array}\right) \frac{\varepsilon}{\pi} I_{l}\left[0, \theta_{H}, \Omega, \zeta \Lambda_{l, n} n+1\right]
\end{aligned}
$$

\begin{tabular}{|c|c|c|c|c|}
\hline Modulation & $\varepsilon$ & $\theta_{H}$ & $\Omega$ & $\zeta$ \\
\hline$M_{c}-\mathrm{DPSK}$ & 1 & $\pi-\pi / M_{c}$ & $\cos \left(\pi / M_{c}\right)$ & $\sin ^{2}\left(\pi / \overline{M_{c}}\right)$ \\
\hline $\begin{array}{l}\text { Correlated Binary } \\
\text { Signals } \\
\text { ( } 9: \text { magnitude of } \\
\text { cross correlation } \\
\text { between the two } \\
\text { signals) }\end{array}$ & $1 / 2$ & $\pi$ & -9 & $\frac{1-9^{2}}{2}$ \\
\hline 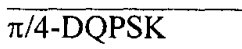 & $1 / 2$ & $\pi$ & $-1 / \sqrt{2}$ & 1 \\
\hline
\end{tabular}

where

$$
\begin{gathered}
I_{1} \cdot\left(\theta_{L}, \theta_{l}, c, a, b\right)=\left(\theta_{L}-\theta_{L}\right)+2 \sum_{n=1}^{b}\left(\begin{array}{l}
b \\
n
\end{array}\right)\left(\frac{a}{c-a-1}\right)^{n} \\
\times \sum_{r=0}^{n} \sqrt{\frac{1+a-c}{1+a+c}}\left(\begin{array}{c}
n-1 \\
r
\end{array}\right)\left(\frac{-2 c}{1+a+c}\right)^{n} \\
\times P\left[\sqrt{\frac{1+a-c}{1+a+c}} \tan \frac{\theta_{L}}{2}, \sqrt{\frac{1+a-c}{1+a+c}} \tan \frac{\theta_{u}}{2}, 1+r\right]
\end{gathered}
$$

for integer $b \geq 1$ and coefficients $\varepsilon, \theta_{H}, \Omega$ and $\zeta$ for these modulation formats are tabulated below.
D. $M_{c}-Q A M$ and $Q P S K$

$$
\begin{aligned}
\bar{P}_{e}= & \sum_{l=0}^{L-k} \sum_{i=1}^{D_{l}} \mu_{i}^{\left(D_{i}-k\right)}\left[\frac{\bar{\gamma}\left(1-\rho^{2}\right)}{\Lambda_{l}}\right]^{k-1 k-1} \sum_{n=0}\left[\frac{M \rho^{2}}{\left(1-\rho^{2}\right)(l+M)}\right]^{\prime \prime} \\
& \times\left(\begin{array}{c}
k-1 \\
n
\end{array}\right) \frac{4 q}{\pi}\left\{I_{s}\left[0, \pi / 2, \frac{3 \Lambda_{l}}{2\left(M_{c}-1\right)}, n+1\right]\right. \\
& \left.-q I_{S}\left[0, \pi / 4, \frac{3 \Lambda_{l}}{2\left(M_{c}-1\right)}, n+1\right]\right\}
\end{aligned}
$$

where $q=1-1 / \sqrt{M}_{\mathrm{c}}$. Noting that signal constellations for 4-QAM and QPSK are virtually identical, the ASER for QPSK may also be obtained by substituting $M_{c}=4$ in (14). However, a more concise solution is given in (10).

E. Noncoherent $M_{c}-F S K$

$$
\begin{aligned}
\bar{P}_{e}= & \sum_{v=1}^{M_{c}-1} \frac{(-1)^{v+1}}{v+1}\left(\begin{array}{c}
M_{c}-1 \\
v
\end{array}\right) \phi_{\gamma}\left(\frac{v}{v+1}\right) \\
= & \sum_{v=1}^{M_{c}-1} \frac{(-1)^{v+1}}{v+1}\left(\begin{array}{c}
M_{c}-1 \\
v
\end{array}\right) \sum_{i=0}^{L-\lambda} \sum_{k=1}^{D_{t}} \mu_{l}^{\left(D_{t}-k\right)}\left[\frac{v+1}{v\left(1+\Lambda_{l}\right)+1}\right] \\
& \times\left[\frac{\bar{\gamma}}{\Lambda_{l}}\left(1-\rho^{2}\right)+\frac{M \rho^{2}(v+1) \bar{\gamma}}{\Lambda_{l}(l+M)\left(v\left(1+\Lambda_{l}\right)+1\right)}\right]^{k-1}
\end{aligned}
$$

It is also apparent that (15) reduces to (11) when $M_{c}=2$ (noncoherent detection), as expected.

We conclude this section by noting that our development rely on the fact that MGF (5) has a similar form to those in [17] and because of the availability of closed-form solutions for the generic trigonometric integrals $I_{S}(, \ldots, .$,$) and$ $I_{l}(., ., ., .,$.$) derived therein.$

\section{OUTAGE PROBABILITY AND MEAN COMBINED SNR}

Outage rate of error probability (hereafter, simply referred as outage probability) is another useful performance measure of diversity systems and this metric quantifies the probability that the instantaneous symbol error probability of the system exceeds a specified value (say $P_{e}{ }^{*}$ ). Hence, the outage probability $P_{o m}$ is given by the CDF of combiner output SNR (4) evaluated at threshold SNR/symbol $\gamma^{*}$ (a coefficient that is dependent on the modulation format and detection scheme), viz.,

$$
P_{o u t}=F_{\gamma}\left(\gamma^{*}\right)
$$

where $\gamma^{*}$ can be obtained by solving $P_{e}\left(\gamma^{*}\right)=P_{t}{ }^{*}$ and $P_{e}($.$) corresponds to the symbol error rate in an AWGN$ (i.e., nonfading) channel. Closed-form solution for $\gamma^{*}$ is available for several common modulation schemes and they are summarized in Table 1. 
Table 1. Threshold SNR/symbol $\gamma^{*}$ in closed-form for several common modulation schemes.

\begin{tabular}{ll}
\hline Modulation/Detection & Threshold SNR/symbol $\gamma^{*}$ \\
\hline BPSK & {$\left[\operatorname{erfc}^{-1}\left(2 P_{e}^{*}\right)\right]^{2}$} \\
CFSK & $2\left[\operatorname{erfc}^{-1}\left(2 P_{e}^{*}\right)\right]^{2}$ \\
NCFSK & $-2 \ln \left(2 P_{e}^{*}\right)$ \\
DPSK & $-\ln \left(2 P_{e}^{*}\right)$ \\
QPSK & $2\left\{\operatorname{erfc}^{-1}\left[2\left(1-\sqrt{1-P_{e}^{*}}\right)\right]\right\}^{2}$ \\
$M_{c}-$ QAM & $\frac{2\left(M_{c}-1\right)}{3}\left(\operatorname{erfc}^{-1}\left(\frac{1-\sqrt{1-P_{e}^{*}}}{1-1 / \sqrt{M_{c}}}\right)\right)^{2}$ \\
MSK & $2\left\{\operatorname{erfc}^{-1}\left[2\left(1-\sqrt{1-P_{e}^{*}}\right)\right]\right\}^{2}$ \\
coherent detection of \\
differentially detected \\
BPSK
\end{tabular}

Next we will derive a simple expression for the average combined SNR at the output of an imperfect SC/MRC receiver by utilizing the PDF or the MGF of SNR. Since the average combined SNR $\bar{\gamma}_{g s c}$ is the first moment (mean) of the random variable $\gamma$, we obtain

$$
\begin{aligned}
\bar{\gamma}_{g, c} & =\int_{0}^{\infty} \gamma p_{\gamma}(\gamma) d \gamma=\sum_{l=0}^{L-A} \sum_{k=1}^{D_{l}} \frac{\mu_{l}^{\left(D_{l}-k\right)}}{\Lambda_{l}^{k-2}}\left[\bar{\gamma}\left(1-\rho^{2}\right)\right]^{k-1} \\
& \times \sum_{n=0}^{k-1}(n+1)\left(\begin{array}{c}
k-1 \\
n
\end{array}\right)\left[\frac{M \rho^{2}}{\left(1-\rho^{2}\right)(l+M)}\right]^{\prime \prime}
\end{aligned}
$$

Alternatively, the first moment can be computed by differentiating the MGF with respect to variable $s$ and then evaluating the derivative at $s=0$ :

$$
\begin{aligned}
\bar{\gamma}_{s, c}= & -\left.\frac{\mathrm{d}}{\mathrm{d} s} \phi_{Y}(s)\right|_{s=0}=\sum_{t=0}^{L-1 /} \sum_{k=1}^{D_{i}} \frac{\mu_{l}^{\left(D_{i}-k\right)}}{\Lambda_{t}^{k-2}}\left[\bar{\gamma}\left(1-\rho^{2}\right)\right]^{k-1} \\
& \times \sum_{n=0}^{k-1}(n+1)\left(\begin{array}{c}
k-1 \\
n
\end{array}\right)\left[\frac{M \rho^{2}}{\left(1-\rho^{2}\right)(l+M)}\right]^{n}
\end{aligned}
$$

which is identical to (17). For the special case of $\rho=1$ (i.e., ideal SC/MRC), the above expression agrees with those presented in [8] and [9] numerically.

\section{SOME IMPORTANT LIMITING CASES}

In this section, we will examine the limiting cases of the correlation coefficient (i.e., $\rho=1$ ) and $\rho=0$ ) and show that our expressions collapses to well-known expressions in the literature. In Section V of [15], we have also carefully examined two other important limiting cases of interest: (a) $M=L$ (MRC); (b) $M=1$ (SC). Specifically, the results for $\mathrm{SC}$ limiting case with imperfect combining appears to be new.

Before proceeding further, we would like to point out that the MGF (5) can re-written more concisely as (by grouping the last summation term in (5) using the binomial expansion identity $\left.(x+y)^{\prime \prime}=\sum_{k=0}^{n}\left(\begin{array}{l}n \\ k\end{array}\right) x^{\mu-k} y^{k}\right)$ :

$$
\phi_{r}(s)=\sum_{l=0}^{L-1 L} \sum_{k=1}^{D_{l}} \frac{\mu_{l}^{\left(D_{l}-k\right)}}{\left(1+s \Lambda_{l}\right)}\left[\frac{\bar{\gamma}}{\Lambda_{l}}\left(1-\rho^{2}\right)+\frac{\xi_{l} \rho^{2}}{\Lambda_{l}\left(1+s \Lambda_{l}\right)}\right]^{k-1} .
$$

or equivalently,

$$
\phi_{\gamma}(s)=\sum_{k=1}^{M} \frac{\mu_{0}^{(M-k)}\left[\left(1-\rho^{2}\right) s \bar{\gamma}+1\right]^{k-1}}{(1+s \bar{\gamma})^{k}}+\sum_{i=1}^{L-1 /} \frac{\mu_{i}^{(1)}}{1+s \bar{\gamma}\left[\frac{M+l\left(1-\rho^{2}\right)}{l+M}\right]}
$$

where $\xi_{1}=\frac{\bar{y} M}{l+M}$ and $\Lambda_{l}=\bar{\gamma}\left[\frac{M+l\left(1-\rho^{2}\right)}{l+M}\right]$.

The above forms allow us to examine the limiting cases more conveniently.

A. Case $\rho=1$

Letting $\rho=1$ (coherent combining with perfect channel gain estimates) in (19), we get

$$
\phi_{y}(s)=\sum_{i=0}^{L-M} \sum_{k=1}^{D_{l}} \mu_{l}^{\left(D_{l}-k\right)}\left(\frac{1}{1+s \xi_{l}}\right)^{k}=\prod_{i=0}^{L-M}\left(\frac{1}{1+s \xi_{l}}\right)^{D_{l}}
$$

by noting that $\mu_{1}^{\left(D_{i}-k\right)}$ is the coefficient of the partial fraction expansion, i.e.,

$$
\mu_{l}^{\left(D_{i}-k\right)}=\left.\frac{\xi_{l}^{k-D_{1}}}{\left(D_{l}-k\right) !} \frac{\partial^{\left(D_{i}-k\right)}}{\partial t}\left[\prod_{i=0, i \neq 1}^{L-\lambda t}\left(\frac{1}{1+\xi_{i}}\right)^{D_{i}}\right]\right|_{i=-1 / \xi_{t}}
$$

Eq. (21) agrees with that of [9, Eq. (12)] after using a variable substitution to change the limits of the product term:

$$
\phi_{\gamma}(s)=\frac{1}{(1+s \bar{\gamma})^{M-1}} \prod_{l=0}^{L-M} \frac{1}{1+\frac{s \bar{\gamma} M}{l+M}}=(1+s \bar{\gamma})^{1-M} \prod_{l=M l}^{L} \frac{1}{1+\frac{s \bar{\gamma} M}{l}} \text { (23) }
$$

(a) MRC

Further substituting $M=L$ in (21), we obtain a familiar expression for the MGF of SNR at the MRC combiner output with $L$ i.i.d. diversity branches in Rayleigh fading because $\mu_{0}^{(0)}=1$ and $\mu_{0}^{(L-k)}=0$ for all $k=1,2, \ldots, L-1$.

(b) $\mathrm{SC}$

Now letting $M=1$ in (21), we get the well known expression for MGF of SNR at the SC combiner output:

$$
\phi_{\gamma}(s)=\sum_{l=0}^{L-1} \mu_{l}^{(0)}\left(\frac{l+1}{l+1+s \bar{\gamma}}\right)=\sum_{l=0}^{L-1}(-1)^{\prime}\left(\begin{array}{c}
L \\
l+1
\end{array}\right)\left(\frac{l+1}{l+1+s \bar{\gamma}}\right)
$$

since $\mu_{1)}^{(0)}=L, \mu_{l}^{(0)}=(-1)^{\prime}\left(\frac{L}{l+1}\right)\left(\begin{array}{c}L-1 \\ l\end{array}\right)$ if $l=1,2, \ldots, L-1$.

Similarly, substituting $\rho=1$ in (1) and (4), we get

$$
\begin{aligned}
p_{\gamma}(\gamma)= & \sum_{k=1}^{M} \frac{\mu_{0}^{(M-k)} \gamma^{k-1}}{(k-1) ! \bar{\gamma}^{k}} \exp \left(-\frac{\gamma}{\bar{\gamma}}\right) \\
& +\sum_{i=1}^{L-A} \frac{\mu_{l}^{(0)}(l+M)}{\bar{\gamma} M} \exp \left(-\frac{\dot{\gamma}(l+M)}{\bar{\gamma} M}\right) \\
F_{\gamma}(\gamma)= & 1-\sum_{k=1}^{M \prime} \mu_{i}^{(M-k)} \exp \left(-\frac{\gamma}{\bar{\gamma}}\right) \sum_{i=0}^{k-1} \frac{1}{w !}\left(\frac{\gamma}{\bar{\gamma}}\right)^{w} \\
& -\sum_{i=1}^{L-1 /} \mu_{i}^{(0)} \exp \left[-\frac{\gamma(l+M)}{\bar{\gamma} M}\right]
\end{aligned}
$$

because all the terms in the last sum equal to zero except when $n=k-1$. These formulas are equivalent to those found in [9, Eq. (16)] and [9, Eq. (24)].

Letting $\rho=1$ in (18) and after simplifications, we obtain

$$
\bar{\gamma}_{g x c}=\sum_{l=0}^{l-M} \sum_{k=1}^{D_{i}} \mu_{l}^{\left(D_{t}-k\right)} \frac{k \bar{\gamma} M}{l+M}=\sum_{k=1}^{M} \mu_{0}^{(M t-k)} k \bar{\gamma}+\sum_{l=1}^{L-M} \mu_{l}^{(i)} \frac{\bar{\gamma} M}{l+M}
$$

which agrees with an equivalent expression found in [8]. 


\section{B. Case $\rho=0$}

On the other extreme case in which the gain estimates are completely uncorrelated with the actual branch gains, the MGF of the SC/MRC combiner output SNR (19) reduces to

$$
\begin{gathered}
\phi_{Y}(s)=\sum_{i=0}^{L-1 /} \sum_{k=1}^{D_{l}} \frac{\mu_{l}^{\left(D_{l}-k\right)}}{(1+s \bar{\gamma})}=\frac{1}{(1+s \bar{\gamma})} \\
\text { since } \sum_{i=0}^{L-N /} \sum_{k=1}^{D_{l}} \mu_{l}^{\left(D_{l}-k\right)}=1 .
\end{gathered}
$$

It is apparent that there will not be any diversity advantage for this particular case because the diversity receiver performance will be dictated by choosing and combining the diversity branches in random. Therefore, the ASER performance will be equivalent to a single channel reception (independent of both $L$ and $M$ ), which is physically intuitive. From this observation, it can be conjectured that there exists an optimal value for the diversity order in energy limited communications which minimizes the ASER (for a specified average SNR per symbol and correlation coefficient) because the combination losses could outweigh the benefits obtained by having additional diversity branches (i.e., in this scenario, the average SNR/symbol per branch is reduced with a higher order of diversity to maintain a fixed energy).

It is also straight-forward to show that (1) and (4) simplifies to the PDF and CDF of SNR with a single channel reception:

$$
\begin{aligned}
& p_{\gamma}(\gamma)=\sum_{i=0}^{L-\Lambda I} \sum_{k=1}^{D_{i}} \mu_{i}^{\left(D_{i}-k\right)} \frac{1}{\bar{\gamma}} \exp \left(-\frac{\gamma}{\bar{\gamma}}\right)=\frac{1}{\bar{\gamma}} \exp \left(-\frac{\gamma}{\bar{\gamma}}\right) \\
& F_{\gamma}(\gamma)=1-\sum_{i=0}^{L-M} \sum_{k=1}^{D_{l}} \mu_{i}^{\left(D_{i}-k\right)} \exp \left(-\frac{\gamma}{\bar{\gamma}}\right)=1-\exp \left(-\frac{\gamma}{\bar{\gamma}}\right)
\end{aligned}
$$

Finally, the average combined SNR (18) collapses to $\bar{\gamma}_{g: c}=\bar{\gamma}$ as expected.

\section{CONCLUSIONS}

This paper derives new, concise expressions for the PDF, $\mathrm{CDF}$ and MGF of SNR at the output of an imperfect $M / L-S C / M R C$ combiner (i.e., with noisy channel gain estimates) operating in a Rayleigh fading environment. The applications of these statistics for characterizing the performance of diversity systems in terms of ASER, outage rate of error probability and average combined SNR are also presented. The MGF is used to derived closed-form ASER expressions for a multitude of digital modulation schemes in conjunction with a "family" of receiver structures (with $\mathrm{SC}$ and MRC as limiting cases). The outage probability can be computed readily using the $\mathrm{CDF}$, and a simple closed-form expression for computing the mean combined SNR may be derived from the PDF or the MGF.

\section{REFERENCES}

[1] N. Kong, T. Eng and L. B. Milstein, "A Selection Combining Scheme for Rake Receivers;" Proc. IEEE ICUPC'95, pp. 426-430, Oct. 1995

[2] T. Eng, N. Kong and L. B. Milstein, "Comparison of Diversity Combining Techniques for Rayleigh Fading Channels," IEEE Trans. on Communications, Vol. 44, pp. 1117-1129, Sept. 1996.
[3] K. J. Kim, S. Y. Kwon, E. K. Hong and K. C. Whang, "Comments on Comparison of Diversity Combining Techniques for Rayleigh Fading Channels" IEEE Transactions on Communications, Vol. 46, pp. 1109-1110, Sept. 1998.

[4] R. Wong, A. Annamalai and V. K. Bhargava, "Evaluation of Predetection Diversity Techniques for Rake Receivers," Proc. IEEE PACRIM'97, pp. 227-230, Aug. 1997.

[5] M. -S Alouini and M. K. Simon, "Performance of Coherent Receivers with Hybrid SC/MRC over Nakagami-m Fading Channels," IEEE Trans. on Vehicular Technology, Vol. 48, pp. 1155-1164, July 1999.

[6] M. -S. Alouini and M. K. Simon, "Application of the Dirichlet Transformation to the Performance Evaluation of Generalized Selection Combining over Nakagami-m Fading Channels," Journal of Communications and Networks, Vol. 1, pp. 5-13, Mar. 1999.

[7] A. Annamalai, C. Tellambura and V. K. Bhargava, "Analysis of Hybrid Diversity Systems on Fading Channels," Proc. IEEE ISWC'99, pp. 70-71, June 1999.

[8] M. Win and J. Winters, "Analysis of Selection/Maximal-Ratio Combining in Rayleigh Fading," Proc. IEEE ICC'99, pp. 6-10, June 1999.

[9] M. -S. Alouini and M. K. Simon, "An MGF-Based Performance Analysis of Generalized Selection Combining over Rayleigh Fading Channels," IEEE Trans. on Communications, pp. 401-414, March 2000.

[10] P. Bello and B. D. Nelin, "Predetection Diversity Combining with Selectively Fading Channels," IRE Transactions on Communica tion Systems, Vol. 10, pp. 32-42, Jan. 1962.

[11] M. Schwartz, W. R. Bennett and S. Stein, Communication Systems and Techniques, McGraw-Hill, New York: 1966

[12] J. G. Proakis, "Probabilities of Error for Adaptive Reception of M-phase Signals," IEEE Transactions on Communication Technology, Vol. 16, pp. 71-81, Jan. 1968.

[13] M. J. Gans, "The Effect of Gaussian Error in Maximal Ratio Combiners," IEEE Transactions in Communication Technology, Vol. 19, pp. 492-500, Aug. 1971.

[14] B. Tomiuk, N. C. Beaulieu and A. Abu-Dayya, "General Forms for Maximal Ratio Diversity with Weighting Errors," IEEE Transactions on Communications, Vol. 47, pp. 488-492, Apr. 1999.

[15] A. Annamalai Jr. and C. Tellambura, "Analysis of Hybrid Selection/Maximal-Ratio Diversity Combiners with Gaussian Errors," submitted to the IEEE Trans. on Communications.

[16] C. Tellambura, A. J. Mueller and V. K. Bhargava, "Analysis of $M$-ary Phase-Shift-Keying with Diversity Reception for Land-Mobile Satellite Channels," IEEE Transactions on Vehicular Technology, Vol. 46, pp. 910-922, Nov. 1997.

[17] A. Annamalai and C. Tellambura, "Error Rates for Nakagami-m Fading Multichannel Reception of Binary and M-ary Signals," to appear in the IEEE Transactions on Communications, 2000 